CIRCUMSTELLAR SHELLS AND ENVELOPES 


\title{
IRAS RESULTS ON CIRCUMSTELLAR SHELLS
}

\author{
H.J. Habing \\ Sterrewacht \\ Leiden.
}

\begin{abstract}
The IRAS Point Source Catalog contains tens of thousands of stars with circumstellar shells. Most are cool stars surrounded by dust-rich envelopes, but many $B$ and A stars are present with ionized circumstellar envelopes. IRAS and its main products are briefly discussed.
\end{abstract}

\section{INTRODUCTION}

In early 1983 a satellite, IRAS, was launched to make a survey

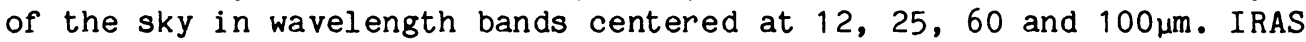
was the first astronomical infrared satellite and, probably, the first satellite to be cryogenically cooled. Its main instruments performed better than expected, and there were hardly any failures until on November 22, 1983 the last drops of coolant (super fluid Helium) were used and the instruments stopped functioning. During the 300 days of satellite operations $95 \%$ of the sky has been covered twice and $72 \%$ for a third time. The data were analysed and the first results were released in November 1984, less than a year after the last observations. One of the two prime "data products" is the Point Source Catalog (IRAS, 1984), containing 245,839 point sources with a reliable detection in at least one of the four wavelength bands. Most point sources have been detected at the two shortest wavelengths, 12 and $25 \mu \mathrm{m}$, and most of these are stars; for approximately 83,000 objects enough information is available to conclude that the infrared emission is from a circumstellar shell. The IRAS Point Source Catalog may well contain the largest collection of circumstellar shells. Therefore it is highly appropriate that at this symposium some time is spent on this collection and on the instrument that produced it. 
TABLE 1.

\section{IRAS - the InfraRed Astronomical Satellite}

Project of the United States of America, the Netherlands and the United Kingdom.

\section{Primary Aim}

A complete and reliable all-sky survey in broad bands at 12, 25, 60 and $100 \mu \mathrm{m}$, plus spectrophotometry between 6 and $23 \mu \mathrm{m}$ of all isolated, bright sources.

\section{Instrument and Performance}

A $60 \mathrm{~cm}$ cryogenically cooled telescope. Angular resolution between $0.8 \times 4.5$ arcmin $^{2}$ and $3.0 \times 5.0$ $\operatorname{arcmin}^{2}(D / \lambda=50,000$ to 6,000$)$. Broadband spectral

resolution $\lambda / \Delta \lambda=1.7$ to 3.2. Spectrophotometry with $\lambda / \Delta \lambda$ varying from 14 to 35 .

\section{Operations}

Three hundred days in 1983. Observations made at time $t$ were repeated at $t+2$ weeks and (for $72 \%$ of the sky) at $t+6$ months. Ninety six percent of the sky has been observed at least twice.

\section{Results}

First generation "data products" released in November 1984. A second generation is being prepared in the U.S.A. and in the Netherlands. The Point Source Catalog has 250,000 entries, of which $2 / 3$ are stars. Approximately 81,000 stars have circumstellar shells.

Positions are (practically) always more accurate than 10 arcsec; the photometric accuracy is expected to be better than $10 \%$. The goals in completeness and reliability have been met.

\section{Some useful references}

-IRAS, the Explanatory Supplement, 1984, eds. C. Beichman, G. Neugebauer, H.J. Habing, P.E. Clegg, T. Chester, U.S. Government Printing office (in press)

-"Light on dark matter", proceedings $1^{\text {st }}$ IRAS conference,ed. F.P. Israël

(Reidel publ., 1986)

-"IRAS Far-Infrared colours of normal stars"

L.B.F.M. Waters, J. Coté, H.H. Aumann, 1986, Astron. Astrophys. (in press)

-"The brightest high-latitude 12 micron IRAS sources"

P. Hacking, G. Neugebauer and 11 other authors, Publ, Astron. Soc. Pac. 97, 616 


\section{THE SATELLITE, ITS MISSION AND ITS MAIN PRODUCTS}

In table 1 I give a short summary of the most important aspects of the IRAS project and what it produced. The project was planned and carried out as a survey mission: its goal was an index of the infrared sky and this was envisioned to be a catalogue of point sources. Consequently a strong emphasis was placed on this catalogue during the years of preparation and during the mission and the phase of data reduction. The goals were high: the catalogue should be as complete as possible (no sources missing) and reliable (all sources included should be real). Such criteria are somewhat contradictory, but nevertheless the resulting catalogue fulfills the two criteria quite well, except at low galactic latitudes (below about $2^{\circ}$; near the galactic centre below $4^{\circ}$ ); there the source density is very high and the detectors could no longer separate individual objects (confusion). For a thorough discussion of completeness, reliability and confusion and how much has been achieved I refer to the Explanatory Supplement (IRAS, 1984).

The IRAS Point Source Catalog is made available on magnetic tape or on microfiche. A printed version is foreseen. Mandatory for a good use is the Explanatory Supplement (IRAS, 1984), which describes the instrument, the observations and the data reduction.

Each point source is characterized by a position and by measured values (or upper limits) for the flux densities in the four wavelength bands. Very good accuracy (usually better than 10 arcsec) was obtained for the positions. Photometric accuracy was more difficult to achieve and, worse, the photometry can not be tested by an independent comparison: for most objects only IRAS data are available, except at $12 \mu \mathrm{m}$. Nevertheless, the photometry is thought to be reliable to about $10 \%$. A revised version of the Point Source Catalog with corrections will appear in late 1986. Also in late 1986 a new catalogue will appear that contains point sources found in small fields from the so called "Additional Observations" program. These fields were scanned by IRAS more often than during the regular survey and consequently much fainter point sources are recognized. The new catalogue will be of special interest for extragalactic studies but occasionally it may prove to be useful for a stellar astronomer.

The Point Source Catalog is reliable and complete and it contains a large number of new sources. Nevertheless the data are rather primitive for the demands of a modern astronomer: angular and spectral resolution are poor (see table 1). Identification of an IRAS source at other wavelengths is of ten necessary before any meaningful interpretation can be started. Luckily, the accuracy of the positions usually makes such an identification possible and unique.

To all who had been involved in the IRAS preparations it came as a happy surprise that the detectors worked so reliably and were so sensitive. It proved to be possible to construct meaningful maps of the brightness distribution - in jargon: "The DC behaviour was excellent". A first generation of sky maps has been made, a second generation is being prepared and the expectation is that here there will be a large improvement in quality. This second generation is 
prepared in the U.S.A. (at IPAC, the IRAS Centre at the California Institute of Technology) and in the Netherlands (at the Space Research Laboratory of the University of Groningen). The two efforts are independent, but the groups remain in contact. Results are to be expected toward the Summmer of 1987. For the subject of this Symposium sky flux maps are of less interest and I will not consider them further.

\section{THE CONTENTS OF THE IRAS POINT SOURCE CATALOG}

The catalogue contains in total 245,839 point sources. The basic data per source are position and flux density (or an upper limit) in each of the four wavelength bands, but additional information is also available (e.g. associations with objects in other catalogues; the presence of confusing sources nearby). Some of the additional information is in a separate file, the Working Survey Data Base (e.g. flux densities from individual detections).

What are these sources? Because the positions are quite accurate (see table 1) identification in other wavelength bands is of ten possible. An example is the Edinburgh program for identification of IRAS point sources near the South Galactic Pole, a first report of which was given by Wolstencroft et al. (1986). (The first part of the definitive report has appeared in Mon. Not. Roy. Astr. 223, 279). Short wavelength and long wavelength sources appear to be very different. Of the 153 sources with a detection at least at $12 \mu \mathrm{m} 149$

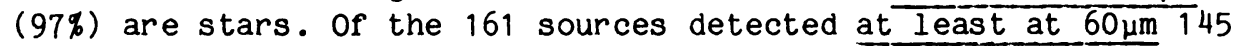
(90\%) are galaxies. At lower galactic latitudes the fraction of stars will increase. The conclusion is that identifiable sources with a $12 \mu \mathrm{m}$ detection are by and large stellar. Are the $12 \mu \mathrm{m}$ sources all identifiable? The brightest ones are as shown by Hacking et al. $(1985)$. They made a study of sources brighter than $[12 \mu]=0.0^{*}$, which corresponds to $28.0 \mathrm{Jy}$; only the 269 sources at $|\mathrm{b}|>30^{\circ}$ were considered (and the Large Magellanic Cloud was avoided). Two of the objects are galaxies, the others are all stars; only a few, rather southernly located sources had no previous identifications. Seventyseven percent of the sources are M-type stars, 9\% are C-stars and $16 \%$ are $\mathrm{K}$-giants. An interesting point, missed by Hacking et al., is that practically all sources identified with a variable star have a colour excess - and vice-versa. I will come back to this point in section 6 .

Another way to study the nature of the point sources is to consider only the IRAS fluxes. Seven percent of the sources has been detected in three bands, so that two colours can be constructed. Figure 1, originally due to Chester (1986), shows the distribution of all point sources detected at 12,25 and $60 \mu \mathrm{m}$ in a colour-colour

* The square brackets stand for "magnitude". Magnitudes remain useful for stellar studies although they are not used in the IRAS Catalog. For their definition and the definition of colours see the appendix. 
diagram of $R_{21}\left(=\log \left[f_{y}(25 \mu \mathrm{m}) / f_{\nu}(12 \mu \mathrm{m})\right]\right)$ and $\mathrm{R}_{32}\left(=\log \left[\mathrm{f}_{\nu}(60 \mu \mathrm{m}) / \mathrm{f}_{\nu} Y(25 \mu \mathrm{m})\right]\right) \stackrel{\mathrm{f}_{\nu}}{\mathrm{f}_{\nu}}$ is the flux density as given in
the catalogue.

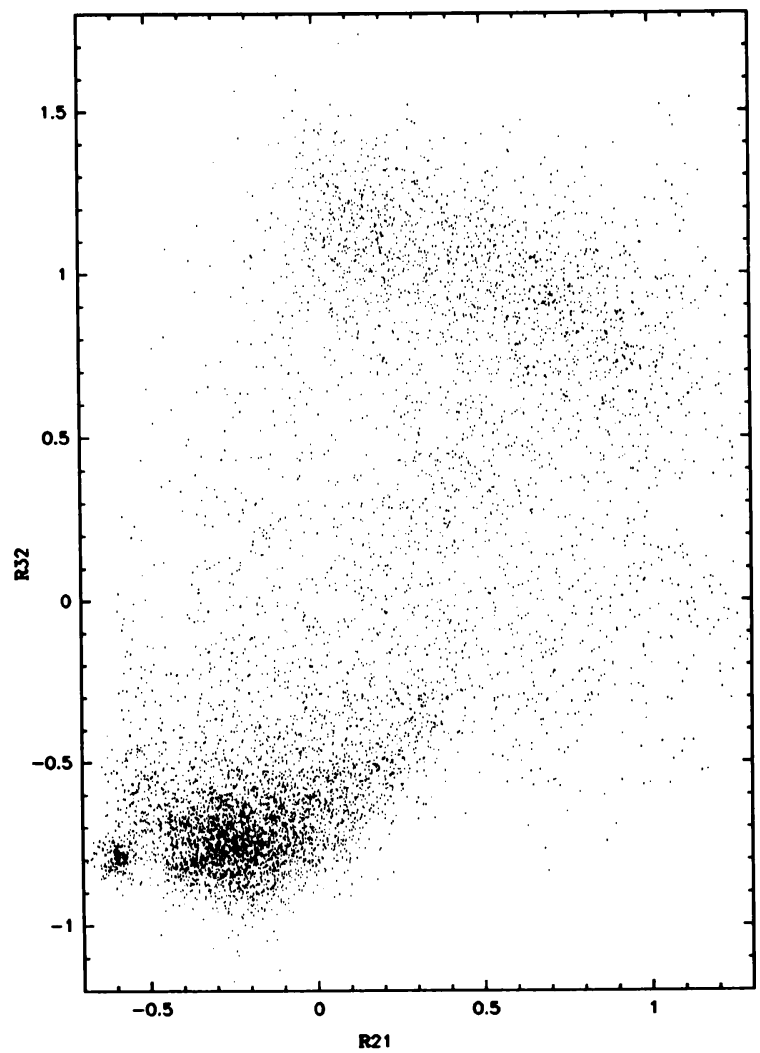

$R 21={ }^{19} \log (\operatorname{mux}(25 \mu \mathrm{m}) / \operatorname{mux}(12 \mu \mathrm{m}))$

$R 32={ }^{19} \log (\operatorname{lux}(60 \mathrm{~mm}) / \operatorname{lux}(25 \mathrm{~mm}))$

Figure 1: Colour-colour diagram of IRAS colours for all objects in the Point Source Catalog with a high-quality detection at 12,25 and $60 \mu \mathrm{m}$.

The flux densities have not been colour-corrected.

There is a sprinkling of points over the whole plane, but there is a strong concentration at lower left and a weaker one at upper right. The "swarm" at the lower left consists of stars with and without circumstellar shells, the other concentration (upper right) consists of galaxies and of "IR sources of galactic origin" (Chester, 1986).

First I discuss the swarm of stars. If all stellar photospheres 
were black bodies and their temperatures over 2000k, then all stellar points would be close to $R_{21}=-0.64$ and $R_{32}=-0.76$ (the RayleighJeans point). Indeed around that point a concentration is present within the stellar swarm. The large swarm to the right (to the east) of the Rayleigh-Jeans point (and well isolated from it) consists of circumstellar dust shells with the $9.7 \mu \mathrm{m}$ band of silicate -thus of cool, oxygen-rich giants with large mass loss rates. This follows from many identifications and from inspection of the Low Resolution Spectra - when available. The gap between the stars with and without circumstellar shells is real; there are no observational limitations known that could have produced the gap (Hacking et al.; 1985). Presumably the presence of the gap means that there is a discontinuity in the distribution of mass loss rates for late type giants. I will come back to the point in section 6. Carbon rich envelopes around $C$-stars tend to have lower $R_{21}$ values and to $l$ ie in the direction north by northeast from the Rayleigh-Jeans point.

Apparently $R_{21}$ is a sensitive indicator for the presence of the $9.7 \mu \mathrm{m}$ silicate band: this band goes into absorption and suppresses the $12 \mu \mathrm{m}$ flux. As a consequence $0-r i c h$ stars are weaker in the $12 \mu \mathrm{m}$ band than C-stars.

Undoubtedly there remain many interesting and peculiar objects to be found in Figure 1 -for example $\alpha$ Lyra (Vega) is at $R_{21}=-0.52 \quad R_{32}=$
0.01 . An interesting area is between $R_{21}=0.3$ and 1.0 , and $R_{32}=-0.6$ 0.01 . An interesting area is between $R_{21}=0.3$ and 1.0 , and $R_{32}=-0.6$
and 0.0 : many planetary nebulae are found there and several objects that may be protoplanetary nebulae ( $v . d$. Veen et al., 1987; Kwok et al., 1987).

The concentration in the upper part of the diagram contains practically all the galaxies with 12,25 and $60 \mu m$ detections in the Point Source Catalog. Here the two colour temperatures, one corresponding to $R_{21}$, the other to $R_{32}$, are very different, indicating that the sources contain both hot and cold dust. This type of spectrum emerges from regions where considerable amounts of matter are heated by hot stars like in starforming regions. Most of the "IR components of galactic origin" in this concentration are probably associated with molecular clouds and star formation processes; they are almost always at very low galactic latitudes.

Qualitatively it thus appears that in all cases the IR point sources consist of clouds of dust reemitting stellar photons. There are two main concentrations in figure 1 because fundamentally two different situations are involved: 1) evolved stars with small amounts of mass (relative to the stellar mass) at very close distance and 2) large clouds of gas and dust heated by embedded or bordering stars; here the dust is at a somewhat larger distance from the star, and the star may have ionized part of the cloud.

For only seven percent of the sources IRAS measured three fluxes, from which two colours can be derived. What about the other sources? Can useful information be extracted without further 1 dentification? The answer is a partial yes for the $39 \%$ sources with two flux density measurements: by using their distribution in the sky, or rather, their distribution with respect to the Galaxy, quite useful information can be extracted. At high declination galaxies 
dominate the Point Source Catalogue at the longer wavelengths. Close to the galactic plane (but just outside it) most 12 and $25 \mu \mathrm{m}$ sources are circumstellar shell objects and $R_{21}$ is an interesting and useful piece of information: see section 6 .

Even for sources detected in only one wavelength band it is sometimes possible to derive their nature: a significant number of point sources is seen only at $100 \mu \mathrm{m}$; almost all are bright spots in the interstellar cirrus, as is proven by the correlation between their position in the sky and the distribution of HI (from $21 \mathrm{~cm}$ line surveys ).

\section{THE ATLAS OF LOW RESOLUTION SPECTRA}

The low resolution spectrograph (see section 2) also functioned without problems. It was a slitless instrument with its dispersion in the direction of the scanning, so that a point source would cast its spectrum gradually over the detectors while the telescope was scanning the sky. This implies that only well isolated point sources yielded useful spectra. Two overlapping wavelength bands, one from 7.7 to $13.4 \mu \mathrm{m}$, the other from 11.0 to $22.6 \mu \mathrm{m}$ were scanned simultaneously. In both cases the resolution increased from 20 at the short wavelength side to 60 at the long wavelength side. The noise varies from (typically) $1.5 \mathrm{Jy}$ at the shorter wavelength to $3 \mathrm{Jy}$ at the longer wavelengths. The atlas of spectra has recently appeared (IRAS, 1986); it contains 5425 spectra of isolated IRAS point sources that are bright at 12 and $25 \mu \mathrm{m}$; five objects are galaxies, the others are (probably) all of galactic origin.

An analysis of the contents of the atlas has been made by olnon (1986). I mainly summarize his results. All the spectra have been classified automatically by computer (a visual inspection afterwards led to changes in only a few percent of the cases). The slope of the spectrum ("red" or "blue") and the strength of spectral features (9.7 $\mu \mathrm{m}$ silicate band; $11.3 \mu \mathrm{m} \mathrm{SiC} \mathrm{band;} \mathrm{ionic} 1$ ines) were used as classification criteria; only information present in the spectrum was used. Altogether there are 9 main classes of objects, each subdivided in 10 subclasses, plus two extra subclasses. To what physical objects do these classes correspond? Ninetyfour percent ( 4735 objects) appear to be stars and most have circumstellar shells; some 1200 objects have very little, if any, circumstellar material around them. The spectra of the shells either contain the silicate bands at $9.7 \mu \mathrm{m}$ (in emission of in absorption) and at $18.8 \mu \mathrm{m}$ (always in emission), or they contain the SiC $11.3 \mu \mathrm{m}$ band indicative of a $\mathrm{C}$-rich shell. The $\mathrm{C}$ spectra are four times less frequent than 0 -(Silicate) spectra. The 685 remaining galactic objects consist of planetary nebulae and of compact HII regions (in about equal proportions) plus a small number of "peculiar stars": proto planetary nebulae, emission line stars. In Figure 2 a sample of spectra of the most common kinds is shown. 


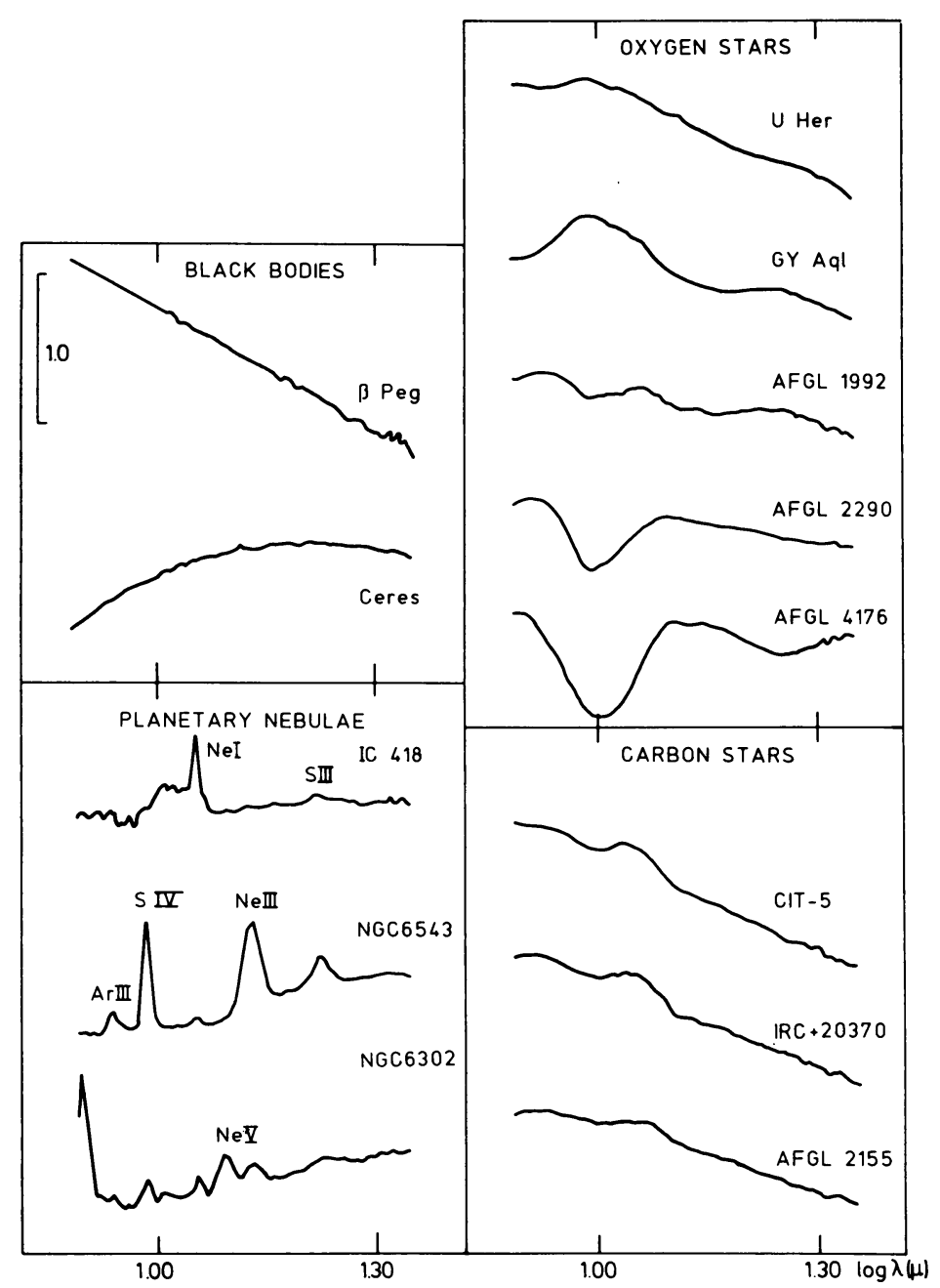

F1gure 2: A sample of low resolution spectra from the Atlas. Among the "black bodies" B Peg is an M2II star with a temperature over a few thousand $K$, and the asteroid Ceres has a temperature around $300 K$. The blackbody spectra are given as a reference.

\section{5. "NORMAL" STARS}

This conference is on circumstellar matter. To make our topic more exclusive I will call stars "normal" if they have no circumstellar matter. IRAS was a poor detector of normal stars, which is not surprising: stellar photospheres are too hot to emit efficiently, even at $12 \mu \mathrm{m}$, the shortest IRAS wavelength. A simple calculation confirms this: normal stars could be detected only when 
they are within a limiting distance $D_{1 i m}$. This distance depends on the detection limit at $12 \mu \mathrm{m}$, on the luminosity $\mathrm{L}$ and on the effective temperature $T_{\text {eff }}$. For a detection limit of $1 \mathrm{Jy}$ values of $D_{1 i m}$ are shown in table 2 as a function of $L$ and $T_{e f f}$ : Main sequence stars and normal giants (luminosity class III) are detected only when they are closer than $1 \mathrm{kpc}$. The last column indicates the number of stars to be detected. The sum of these is much less than the total number of short wavelength sources in the IRAS catalogue. This confirms the conclusion drawn by Olnon (1986) from his analysis of the contents of the LRS-atlas (see section 4): most of the stellar objects have circumstellar shells, only a small fraction (0lnon estimates 1/4) are "normal".

The last colomn can not be very accurate -for example the number of B stars in the Bright Star Catalogue is much less than 3000. Nevertheless qualitatively it gives the right impression: the number of dwarfs decreases with advancing spectral type, and most bright $G$ and $\mathrm{K}$ stars are giants.

TABLE 2.

Limiting distances and expected numbers of normal stars

\begin{tabular}{|c|c|c|c|c|c|c|}
\hline Spectral type & $V-[12 \mu]^{1)}$ & $v_{1 i m}{ }^{2)}$ & $M_{v}^{3)}$ & $\underset{(\text { parsec })}{D_{1 i m}}$ & $\stackrel{\rho}{4)}_{(\text {parsec })^{-3}}$ & $\begin{array}{l}\text { expected } 5 \text { ) } \\
\text { number }\end{array}$ \\
\hline B5 $\mathrm{V}$ & -0.47 & +3.93 & -0.9 & 92 & 0.0009 & 3,000 (?) \\
\hline$A O V$ & +0.05 & +4.45 & +0.7 & $56 ?$ & & \\
\hline A5 $\mathrm{V}$ & +0.53 & +4.93 & +2.0 & $39 \mathrm{~J}$ & 0.001 & 117 \\
\hline FO $\mathrm{V}$ & +0.84 & +5.24 & +2.8 & $31)$ & & \\
\hline F5 V & +1.20 & +5.60 & +3.8 & $23)$ & 0.003 & 250 \\
\hline GO V & +1.50 & +5.90 & +4.6 & $18)$ & 0.004 & 80 \\
\hline G5 V & +1.84 & +6.24 & +5.2 & 16 & & \\
\hline KO V & +2.42 & +6.82 & +6.0 & 15 & 0.009 & 120 \\
\hline G5 III & +2.18 & +6.58 & +1.5 & 104 & 0.0008 & 3700 \\
\hline KO III & +2.48 & +6.88 & +0.8 & $164)$ & 0.0001 & 22,000 \\
\hline K5 III & +3.73 & +8.13 & +0.0 & $423)$ & & \\
\hline
\end{tabular}

1) from Waters et al. (1986)

2) limiting $v$ magnitude for inclusion in the Point Source Catalog,assuming that $[12 \mu]_{11 \mathrm{~m}}=+4.40$

3) from Allen, Astrophysical Quantities, $2^{\text {nd }}$ edition, $\$ 99$

4) local density of stars, from Allen, Astrophysical Quantities, $2^{\text {nd }}$ edition, $\$ 117$

5) assuming a spherical volume, except for the $K$ giants, where a cylinder with a height of $700 \mathrm{pc}$ was assumed and a radius of $320 \mathrm{pc}$ 
What are the colours of normal stars? An illuminating paper is by waters et al. (1986). They consider only IRAS point sources identified with stars from the Bright Star Catalogue (Hoffleit and Jaschek, 1982). This is a sensible approach: the PSC is complete at $12 \mu \mathrm{m}$ for magnitudes brighter than +4.4 . After elimination of doubtful cases Waters et al. have a sample of 6013 stars with a good $12 \mu \mathrm{m}$ detection (and an increasingly smaller number of objects detected in the other IRAS bands).

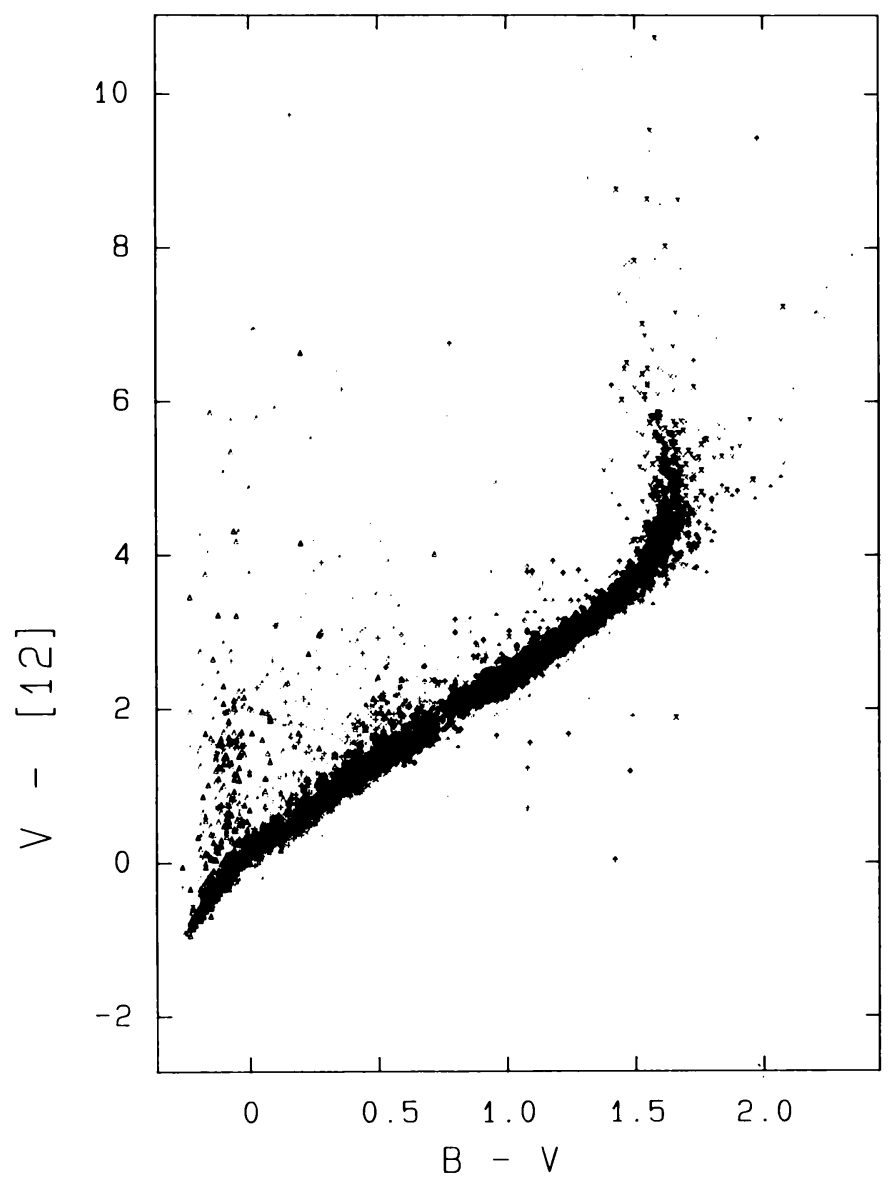

Figure 3: Colour-colour diagram for objects from the Bright Star Catalogue identified with objects in the point Source Catalog (Waters et al, 1986).

Figure 3 shows a diagram of $B-V$ versus $V-[12 \mu]$; the $12 \mu \mathrm{m}$ flux density is converted into a magnitude using $28.0 \mathrm{Jy}$ as the zero point (see appendix). Figure 3 shows at a glance which stars are "normal" and 
where the deviations occur: a narrow, very well defined band runs in the middle of the diagram. There is an obvious gap in the band at B$V=0.75$, which is due to the fact that the number of main-sequence stars drops rapidly for spectral type later than G5. But at $B-V=0.8$ giant stars begin to fill in - this follows also from the last column of table 2. That the stars in the narrow band are "normal" stars follows from a comparison for main sequence 0 , B and A stars (B$\mathrm{V}<0.8$ ) with colours expected from model atmospheres by Kurucz. The theoretical curve has the same shape but is systematically lower by $10 \%$. This shift may have been caused by the assumption of LTE in the model computations.

Almost all deviating cases in Figure 3 are above the band of normal stars. The few cases below can probably be accounted for in terms of duplicity, variability and misidentifications; they are not further considered by Waters et al.. Being situated above the band implies an excess flux at $12 \mu \mathrm{m}$. First consider the excesses for B$V<0.75$. Most excesses occur around $B-V=0.0$, that is for spectral types AO or earlier. Especially among $B$ and $A$ type stars quite a $f$ ew show IR excesses: about $1 / 2$ of the $B$ stars and about $1 / 4$ of the A stars have a $V-[12 \mu]$ excess of 0.2 or larger. The percentage of stars with an excess drops if one goes to later spectral types. Only $5 \%$ of the $\mathrm{K}$ stars in Figure 3 (practically all giants) have a $V-[12 \mu]$ excess larger than 0.2. However, for $M$ stars the situation is completely reversed: Figure 3 shows a large number of excesses beyond $B-V=1.5$. I will discuss this further in the next section.

It thus appears that infrared excess occurs frequently in $M$ giants and in (main sequence) stars of spectral type earlier than A8. Dwarfs and giants with spectral types $F, G$ and $K$ are mostly, but not always free of an excess.

\section{DGE STARS: COO- GIANTS WITH DUSTY ENVELOPES}

As discussed in the previous section only a few percent of the $K$ stars have an infrared excess, but this is not at all true for $M$ stars: Waters et al. state that $55 \%$ of the MO-M2 stars have a $V-[12 \mu]$ excess larger than 0.2 , and they do not even discuss stars of 1 ater spectral type (i.e. B-V>1.6). Clearly, if in a given sample 55\% of the stars have an excess, the question rises: what is the standard, what is normal? Before I attempt to answer I will first try to answer another question: what produces the excess emission?

Already in the mid-sixties it became clear that late-type giants emit more in the infrared than expected from a blackbody extrapolation from shorter wavelengths. Solid particles in a circumstellar envelope were thought to be the cause: they will convert stellar photons very efficiently into infrared photons over a broad range in infrared wavelengths. In oxygen-rich giants the particles were recognized as a kind of silicates when Woolf and Ney (1969) identified the $9.7 \mu \mathrm{m}$ absorption band. Later a band at $11.3 \mu \mathrm{m}$ in the (less frequently found) carbon-rich giants was identified as SiC (Treffers and Cohen, 1974). The same bands are found in the IRAS 
atlas of low-resolution spectra (see section 4): most stars with excess emission have the 9.7 band, a few have the 11.3 band. Some stars have the silicate band in emission, others have it in absorption: clearly the amount of circumstellar matter varies strongly from one star to the other. Theoretical infrared spectra have been calculated with increasing success, starting with the pioneering work by Jones and Merrill (1976) and by Bedijn (1977). For very recent work see Rowan- Robinson et al. (1986) and Bedijn (1986). Theory and observation can be brought together very closely with a minimum of assumptions: see Figure 4.

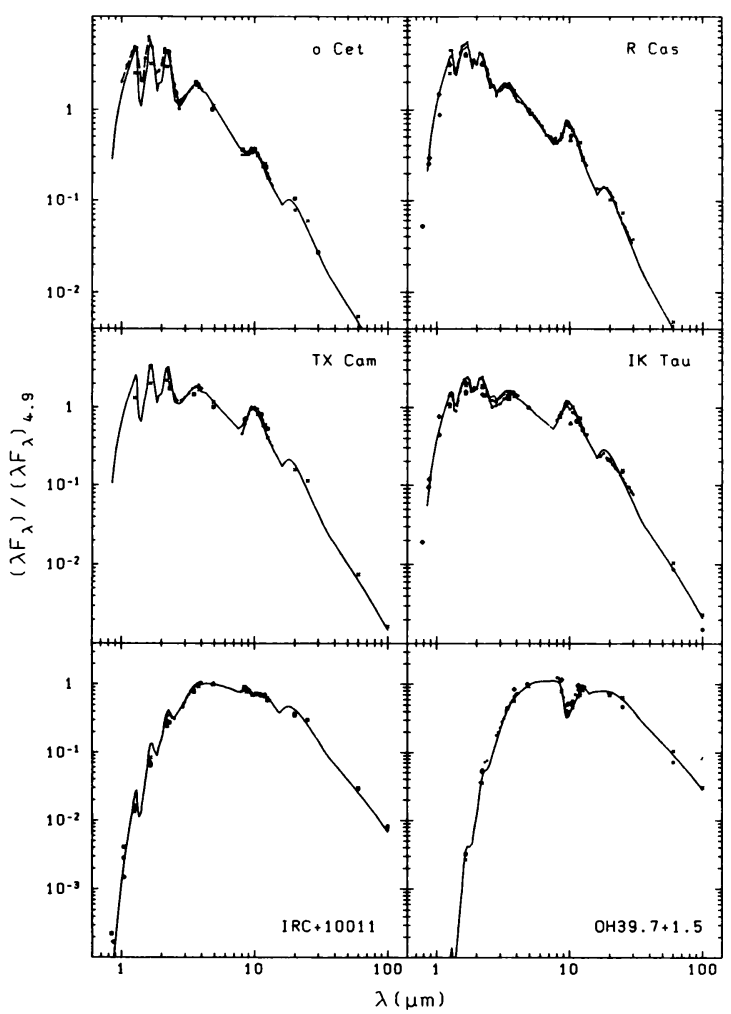

Figure 4: Infrared spectra of long period variables; dots and crosses are observations and the drawn line is a model fit, based on the calculation of the transfer of radiation through a dusty envelope (Bedijn, 1986).

The same basic model is used for all spectra, only the optical depth (measured at 9.7 $\mathrm{m}$ ) varies from $\tau=0.4$ for o Cet to $\tau=10$ for $\mathrm{OH} 39.7+1.5$. 
The main difference between various authors, and the main source of uncertainty, is the precise absorption characteristics of the dust. The IRAS Atlas of Low Resolution Spectra provides a large and homogeneous data base that may clarify the absorption characteristics much better - the work by Papoular, Pegourie and others here at this Symposium is certainly a step in that direction. For a more extensive and useful review see Kwok (1986).

At this point I like to propose some nomenclature. Stars with strong infrared excesses are sometimes called CE-stars (circumstellar envelope stars) and, sometimes, OH/IR stars. Both names are inadequate. The first because it does not distinguish between stars with ionized circumstellar shells, e.g. Be stars, and the cool giants with dusty envelopes. The second is inadequate because only a fraction of the stars show $\mathrm{OH}$ maser emission. I propose to call cool giants with dust-rich envelopes "DGE-stars": dust-gas enveloped stars. The $O H / I R$ stars are then a natural subclass of the DGE-stars. And many $M$ giants will be DGE-stars.

I will now turn again to the question which $M$ giants have IR excess emission and which do not. Let me try to swim in deep water and jump from the diving board of theory. Much work has been done on the interior structure of well evolved stars (much more, so it appears, than on the outer structure) - for a review see Iben and Renzini (1983). This makes it clear that stars of very different initial masses may become $M$ giants; and they may become it more than once. A sample of observed $M$ giants must clearly be a mix of objects with very different masses and internal structures. Two basic structures can be distinguished: (i) stars with degenerate helium cores and extensive hydrogen envelopes burning hydrogen into helium at the boundary between the two; such stars are called First Giant Branch stars (FGB stars); (ii) stars with a core of degenerate carbon and oxygen, surrounded again by an envelope of hydrogen; the two parts are separated by two thin layers, one in which hydrogen burns into helium and one in which intermittently helium burns into carbon. Such stars are called Asymptotic Giant Branch Stars (AGB stars). Most $A G B$ stars become much brighter than FGB stars.

The dichotomy between $F G B$ and $A G B$ stars reminds one of the fact that there is a small group of $M$ giants which are highly variable: Mira variables. Their spatial density is only a few percent of that of the "normal" M giants (Plaut, 1965). Wood (1974) has suggested that Mira variables are AGB stars: their luminosities are high and their spatial motions show that they have main sequence masses $>1.0 M_{G}$. With this in mind I speculate that the non-variable (i.e. non-Mira) $M$ giants are FGB stars, and that the DGE-phenomenon occurs only in highly variable AGB stars. To support my case I will present some evidence that pulsation and mass loss are closely connected. I realize, however, that I cannot make a fully convincing case yet, or answer all objections that might be raised.

The paper by Hacking et al. on the brightest $12 \mu \mathrm{m}$ IRAS sources outside of the galactic plane contains 130 identifications with variable stars. Of these 119 have a $[2.2 \mu]-[12 \mu]$ index larger than 0.4 - value of 0.0 is expected for a blackbody, and, indeed, $\mathrm{K}$-giants 
in the Hacking et al. paper have low values $(<0.2)$. I have selected 10 stars out of these 119 with index $>1.0$, checked their visual amplitude and found in nine cases that this is larger than 1.6; six stars are Mira's, 3 are SRb variables; one object I could not trace. Next consider the 11 variables with a small index $[2.2 \mu]-[12 \mu]$. For six I could find the variability properties; they are all SR or L variables, with visual amplitudo's $<1.0$. This suggests that variables with large excesses have large pulsation amplitudes, and those with small (or no) amplitudes have small or no $[2.2 \mu]-[12 \mu]$ excesses. The Hacking et al. paper also contains $38 \mathrm{M}$ giants with a name that does not indicate variability. Checking them in the Bright Star Catalogue I sometimes find the indication "var?" indicating at most small amplitude variations. Of these 38 only 4 have a colour index larger than 0.4 - the others donot. Again I find the suggestion that small or no pulsations imply a low colour excess.

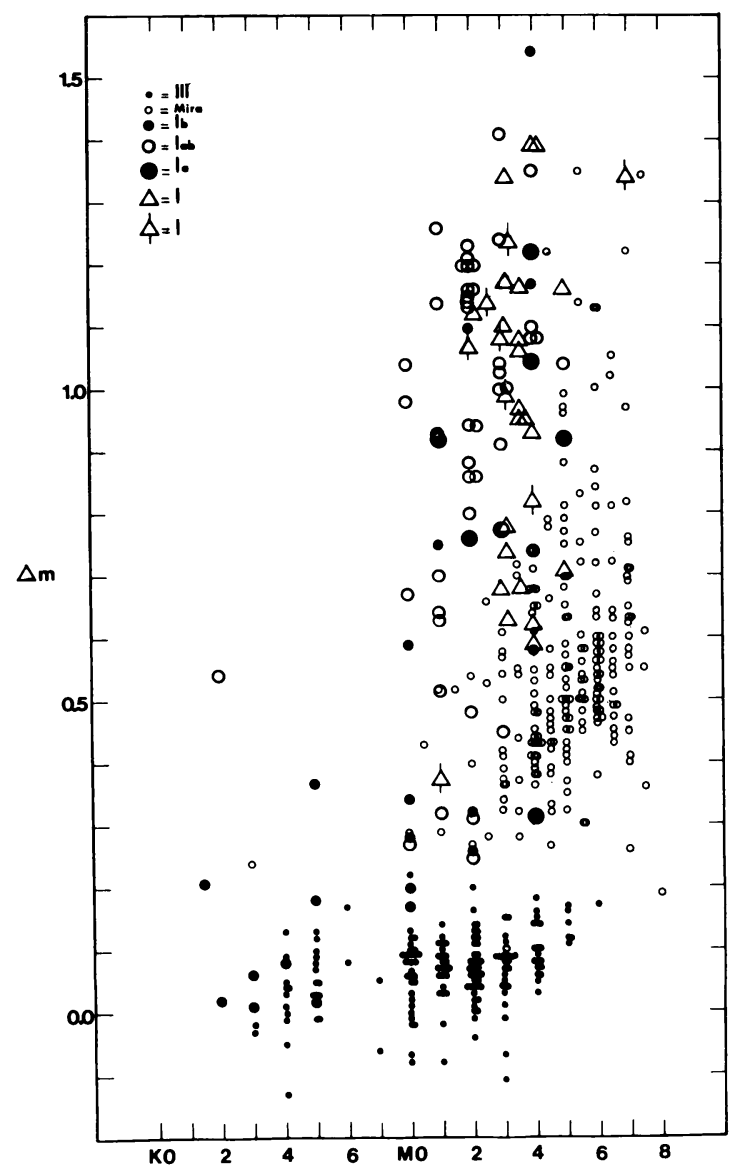

Figure 5: The $[12 \mu]-[25 \mu]$ colour (called " $\Delta M "$ ) of late type giants (luminosity class III) and of supergiants as a function of spectral type (see text). Courtesy of M. Raharto (Lembang). 
The same suggestion is coming from a somewhat different direction. In Figure 5 is shown the $[12 \mu]-[25 \mu]$ index (in the figure it is called " $\Delta \mathrm{m}$ ") for some IRAS point sources for which a good spectral and luminosity classification is available; I owe this figure to M. Raharto from Lembang observatory. It shows very clearly how different classes of objects have different $[12 \mu]-[25 \mu]$ indices: $M$ giants, mostly taken from the Bright Star Catalogue and without variability (or at most: variability with a small amplitude), have a small index. Mira variables and supergiants have a range of index values, but never a small index. There seems to be a gap of about $\Delta m=0.15$ between the two groups. It is probably the same gap that occurs in Figure 1 near the Rayleigh-Jeans Point (see section 3 for some discussion).

This is thus the suggestion: among $M$ giants two fundamentally different types are present: FGB stars with no, or small amplitude variation in the visual and with low IR excesses and AGB stars pulsating with large visual amplitudes (there are mainly Mira variables) and with large IR excesses. The two types occupy wellseparated areas in Figure 5; the separation between the two areas is probably real: AGB stars have always a significant excess, FGB stars do not.

In this section I have discussed the topic of the DGE-stars. Although the interest in this topic began when the first IR observations were made by the pioneers in the sixties, it is obvious to me that IRAS has given a very strong stimulus to the field and may well produce a breakthrough in our understanding of the late stages of evolution. This in itself is an important reason to be interested in DGE stars. There are, however, two more reasons.

The first is that the circumstellar envelopes are very rich in molecules. Carbon star envelopes are already well known for this richness -e.g. IRC +10216 has a large collection of different molecules (see e.8. Johansson et al., 1984), and oxygen-rich stars begin to give away their secrets as well (Guilloteau et al., 1986). To study chemistry under astrophysical conditions these envelopes may be more suitable than molecular clouds in interstellar space: in circumstellar shells one knows more accurately the physical conditions (radiation field, density, temperature).

The second additional reason why DGE stars are interesting is that they are very strong IR emitters (luminosities 2000 $20,000 \mathrm{~L}_{\theta}$, $\mathrm{M}_{\text {bol }}$ between -3.5 and -6.0 ) and that they do not suffer much interstellar extinction. Hence they can be studied at large distances in the Galaxy where optical observations fail. Plotted on a map of the sky DGE stars clearly outline the disk and the bulge of the Galaxy (Habing et al., 1985). The bulge and the disk of the Galaxy are two quite different components of the Galaxy, the first considered to be very old, the other young, or, at least, to be continuously rejuvenated. The IRAS data have given us new and very effective means to study the Galaxy "in a new light". Elsewhere I will review some of the means (Habing, 1987). 


\section{CONCLUSIONS}

For the student of circumstellar matter the IRAS Point Source Catalog contains a very large and new data base with ten thousands of interesting objects. There are many envelopes of ionized material surrounding $B$ and A stars but most objects will be DGE-stars, i.e. stars with dust-rich envelopes. The latest stages of stellar evolution are heavily influenced by mass loss; it is likely that analyses of the DGE-stars discovered by IRAS will shine much light on this process - a possible breakthrough.

One final point. I do not believe in the superiority of large numbers, despite my recent attachement to space research. Personally I have always found (and still find) the single discovery of the excess emission around VEGA ( $\alpha$ Lyra) by Aumann and Gillett (see e.g. Aumann et al., 1984) the most rewarding and interesting of the discoveries by IRAS. Perhaps other discoveries of similar importance are still hidden in the large IRAS database.

\section{APPENDIX}

Magnitudes and colours in the infrared

${ }_{1}$ The magnitude of a point source is defined as -2.5 times the log ratio of the flux density and some standard flux density (the zero point) both at the same wavelength. Magnitudes are symbolized by $[\lambda]$, where $\lambda$ is the wavelength. Traditionally the spectrum of some "average" AO V star was chosen to define zero points at different wavelengths. In the IRAS context this habit has been replaced by the marginally different habit to use a $10,000 \mathrm{~K}$ blackbody viewed at a solid angle of $1.5710^{-16}$ ster. This means that $[12 \mu]=0.0$ corresponds to $28.0 \mathrm{Jy},[25 \mu]=0.0$ to $6.7 \mathrm{Jy}$.

A "colour index" (or "colour" for short) is the difference between magnitudes at two different wavelengths, in the sense "blue minus red". Redder objects have increasing colour indices. In the infrared the colour index for "normal stars" is always very small and increases only marginally with redness. This is a consequence of being on the long wavelength side of the blackbody curve.

\section{REFERENCES}

Allen, C.W. 1963, Astrophysical Quantities ( $2^{\text {nd }}$ ed.), the Athlone Press (London)

Aumann, H.H., Gillett, F.C., Beichman, C.A., de Jong, T., Hovck, J.R., Low, F.J., Neugebauer, G., Walker, R.G., Wesselius, P.R. 1984, Astrophys. J. Lett. 278, L23

Bedijn, P.J. 1977, thesis, Leiden University

Bedijn, P.J. 1986, in "Light on Dark Matter", ed. F.P. Israel, Reidel Publ. Cy., Dordrecht p. 119

Chester, T.J. 1986, in "Light on Dark Matter", ed. F.P. Israel, 
Reidel Publ. Cy., Dordrecht, p.3.

Guilloteau, S., Lucas, R., Nguyen-Q-Rieu, Omont, A. 1986, Astron. Astrophys. 165, L1

Habing, H.J. 1987, in "The Galaxy", ed. G. Gilmore, Reidel Publ. Cy., Dordrecht (in press)

Habing, H.J., Olnon, F.M., Chester, T., Gillett, F., Rowan-Robinson, M., Neugebauer, G. 1985, Astron. Astrophys. 152, L1

Hacking, P., Neugebauer, G., Emerson, J., Beichmann, C., Chester, T., Gillett, F., Habing, H.J., Helou, G., Houck, J., Olnon, F., Rowan-Robinson, M., Soifer, T., Walker, D. 1985, Publ. Astron. Soc. Pac. 97,616

Hoffleit, D., Jaschek, C. 1982, "The Bright Star Catalogue", Yale University Observatory, New Have, Conn., USA

Iben, I., Renzini, A. 1983, Ann. Rev. Astron. Astrophys. 21, 271

IRAS, 1984, "The Explanatory Supplement", eds. C.A. Beichmann, G. Neugebauer, H.J. Habing, P.E. Clegg, T.J. Chester (The United States Government Printing offices).

IRAS, 1986, Atlas of Low Resolution Spectra, eds. F.M. Olnon and E. Raimond, Astron. Astrophys. Suppl. Ser. 65, 607.

Johansson, L.E.B., Andersson, C., Elldér, J., Friberg, P., Hjalmarson, A, Hoegl und, B., Irvine, W.M., Olofsson, H., Rydbeck, G. 1984, Astron. Astrophys. 130, 227

Jones, T.W., Merrill, K.M. 1976, Astroph. J. 209. 509

Kwok, S.Y. 1986, preprint, to appear in "Phys $\overline{i c s}$ Reports"

Kwok, S.Y., Hri vnak, B.J., Milone, E.F. 1986, Astrophys. J. (in press)

Olnon, F.M. 1986, in "Light on Dark Matter", ed. F.P. Israel, Reidel Publ. Cy., Dordrecht, p. 31 .

Plaut, L. 1965, in "Galactic Structure" (Stars and Stellar systems, vol. V), eds. A. Blaauw and M. Schmidt (The University of Chicago Press), p. 267

Rowan-Robinson, M., Lock, T.D., Walker D.W., Harris, S. 1986, Mon. Not. Roy. Astr. Soc. 222, 273

Treffers, R., Cohen, M. 1974, Astrophys. J. 188, 545

Van der Veen, W., Habing, H.J., Geballe, T. $\overline{1987}$, in "Planetary and Protoplanetary Nebulae", ed. A. Preite-Martinez, Reidel Publ.Cy., Dordrecht (in press)

Waters, L.B.F.M., Coté, J., Aumann, H.H. 1986, Astron. Astrophys. (in press)

Wood, P.R. 1974, Astrophys. J. 190, 609

Woolf, N.J., Ney, E.P. 1969, Astrophys. J. Letters 155, L181 